\title{
Existence of Positive Solutions to Singular $p$-Laplacian General Dirichlet Boundary Value Problems with Sign Changing Nonlinearity
}

\author{
Qiying Wei, ${ }^{1}$ You-Hui Su, ${ }^{2}$ Subei $\mathrm{Li}^{2}{ }^{2}$ and Xing-Xue Yan $^{3}$ \\ ${ }^{1}$ College of Sciences, China University of Mining and Technology, Xuzhou, Jiangsu 221008, China \\ ${ }^{2}$ School of Mathematics and Physics, XuZhou Institute of Technology, Xuzhou, Jiangsu 221008, China \\ ${ }^{3}$ Department of Mathematics, Hexi University, Zhangye, Gansu 734000, China
}

Correspondence should be addressed to You-Hui Su, suyouhui@xzit.edu.cn

Received 27 December 2008; Revised 21 February 2009; Accepted 25 February 2009

Recommended by Paul Eloe

By using the well-known Schauder fixed point theorem and upper and lower solution method, we present some existence criteria for positive solution of an $m$-point singular $p$-Laplacian dynamic equation on time scales with the sign changing nonlinearity. These results are new even for the corresponding differential $(\mathbb{T}=\mathbb{R})$ and difference equations $(\mathbb{T}=\mathbb{Z})$, as well as in general time scales setting. As an application, an example is given to illustrate the results.

Copyright (C 2009 Qiying Wei et al. This is an open access article distributed under the Creative Commons Attribution License, which permits unrestricted use, distribution, and reproduction in any medium, provided the original work is properly cited.

\section{Introduction}

Initiated by Hilger in his Ph.D. thesis [1] in 1988, the theory of time scales has been improved greatly ever since, especially in the unification of the theory of differential equations in the continuous case and the theory of finite difference equations in the discrete case. For the time being, it remains active and attracts many distinguished researchers' attention. The reason is two sided. On the one hand, the calculus on time scales not only can unify differential and difference equations, but also can provide accurate information of phenomena that manifest themselves partly in continuous time and partly in discrete time. On the other hand, it is also widely applied to the research of biology, heat transfer, stock market, wound healing and epidemic models [2-6], and so forth. For instance, Hoffacker et al. have used the theory to model how students suffering from the eating disorder bulimia are influenced by their college friends. With the theory on time scales, they can model how the number of sufferers changes during the continuous college term as well as during long breaks [5]. Hence, the dynamic equations on time scales are worth studying theoretically and practically $[3,5,7]$. 
Here and hereafter, we denote $\varphi_{p}(u)$ is $p$-Laplacian operator, that is, $\varphi_{p}(u)=|u|^{p-2} u$ for $p>1$ and $\left(\varphi_{p}\right)^{-1}=\varphi_{q}$, where $1 / p+1 / q=1$. We make the blanket assumption that $0, T$ are points in $\mathbb{T}$, by an interval $(0, T)_{\mathbb{T}}$ we always mean $(0, T) \cap \mathbb{T}$. Other types of interval are defined similarly.

Recent research results indicate that considerable work has been made in the existence problems of solutions of boundary value problems on time scales, for details, see [8-16] and the references therein. In particular, some of them are considered the existence of positive solutions of $p$-Laplacian boundary value problems on time scales, see [17-22]. The main tools used in these papers are the various fixed point theorems in cones. Very recently, when the nonlinear term $f$ is allowed to change sign, Su et al. [23-25] proved the existence of positive solutions to $p$-Laplacian dynamic equations with sign changing nonlinearity on time scales.

Motivated by references [23-25], we consider the following $m$-point singular $p$ Laplacian boundary value problem on time scales of the form

$$
\begin{aligned}
& \left(\varphi_{p}\left(u^{\Delta}(t)\right)\right)^{\nabla}+q(t) f(t, u(t))=0, \quad t \in(0, T)_{\mathbb{T}}, \\
& u(0)=0, \quad u(T)=\sum_{i=1}^{m} \psi_{i}\left(u^{\Delta}\left(\xi_{i}\right)\right), \quad m \in \mathbb{N},
\end{aligned}
$$

where $f(t, u):(0, T)_{\mathbb{T}} \times(0, \infty) \rightarrow \mathbb{R}$ is continuous and $\psi_{i}: \mathbb{R} \rightarrow \mathbb{R}$ are continuous, nondecreasing and $\psi_{i}$ may be nonlinear, $0 \leq \xi_{1}<\xi_{2}<\cdots<\xi_{m} \leq T$. The singularity may occur at $u=0, t=0$ and $t=T$, and the nonlinearity is allowed to change sign. In particular, the boundary condition (1.2) includes the Dirichlet boundary condition. We obtain some new existence criteria for positive solutions of the boundary value problem (1.1) and (1.2) by using the upper and lower method. Our results are new even for the corresponding differential $(\mathbb{T}=\mathbb{R})$ and difference equations $(\mathbb{T}=\mathbb{Z})$, as well as in general time scales setting. As an application, an example is given to illustrate these results. In particular, our results improve and generalize some known results of Agarwal et al. [26], O'Regan [27] $(p=2)$ and Lü et al. [28] when $\mathbb{T}=\mathbb{R}$; include the results of Lü et al. [29] when $\mathbb{T}=\mathbb{R}$; extend and include the results of Jiang et al. [30] in the case of $\mathbb{T}=\mathbb{Z}$.

For the convenience of statements, now we present some basic definitions and lemmas concerning the calculus on time scales that one needs to read this manuscript, which can be found in $[3,7]$. One of other excellent sources on dynamical systems on time scales is from the book in [31].

Definition 1.1 (see $[3,7]$ ). A time scale $\mathbb{T}$ is a nonempty closed subset of $\mathbb{R}$. It follows that the jump operators $\sigma, \rho: \mathbb{T} \rightarrow \mathbb{T}$ defined by

$$
\sigma(t)=\inf \{\tau \in \mathbb{T}: \tau>t\}, \quad \rho(t)=\sup \{\tau \in \mathbb{T}: \tau<t\}
$$

(supplemented by $\inf \varnothing:=\sup \mathbb{T}$ and $\sup \varnothing:=\inf \mathbb{T}$ ) are well defined. The point $t \in \mathbb{T}$ is left-dense, left-scattered, right-dense, right-scattered if $\rho(t)=t, \rho(t)<t, \sigma(t)=t, \sigma(t)>t$, respectively. If $\mathbb{T}$ has a right-scattered minimum $m$, define $\mathbb{T}_{\kappa}=\mathbb{T}-\{m\}$; otherwise, set $\mathbb{T}_{\kappa}=\mathbb{T}$. If $\mathbb{T}$ has a left-scattered maximum $M$, define $\mathbb{T}^{\kappa}=\mathbb{T}-\{M\}$; otherwise, set $\mathbb{T}^{\kappa}=\mathbb{T}$. The forward graininess is $\mu(t):=\sigma(t)-t$. Similarly, the backward graininess is $v(t):=t-\rho(t)$. 
Definition 1.2 (see [7]). We say that a function $f: \mathbb{T} \rightarrow \mathbb{R}$ is right-increasing at a point $t_{0} \in$ $\mathbb{T} \backslash\{\max \mathbb{T}\}$ provided the following conditions hold.

(i) If $t_{0}$ is right-scattered, then $f\left(\sigma\left(t_{0}\right)\right)>f\left(t_{0}\right)$.

(ii) If $t_{0}$ is right-dense, then there is a neighborhood $U$ of $t_{0}$ such that $f(t)>f\left(t_{0}\right)$ for all $t \in U$ with $t>t_{0}$.

Similarly, we say that $f$ is right-decreasing if above in (i), $f\left(\sigma\left(t_{0}\right)\right)<f\left(t_{0}\right)$ and (ii), $f(t)<$ $f\left(t_{0}\right)$.

Definition 1.3 (see [3]). A function $f: \mathbb{T} \rightarrow \mathbb{R}$ is called predifferentiable with (region of differential) $D$ provided the following conditions hold:

(i) $f$ is continuous on $\mathbb{T}$;

(ii) $D \subset \mathbb{T}^{\kappa}$;

(iii) $\mathbb{T}^{\kappa} \backslash D$ is countable and contains no right-scattered elements of $\mathbb{T}$;

(iv) $f$ is differentiable at each $t \in D$.

Next, we list some lemmas which will be used in the sequel.

Lemma 1.4 (see $[3,7])$. Suppose $f: \mathbb{T} \rightarrow \mathbb{R}$ is a function and let $t \in \mathbb{T}^{\kappa}$, then one has the following:

(i) If $f$ is differentiable at $t$, then $f$ is continuous at $t$.

(ii) If $f$ is continuous at $t$ and $t$ is right-scattered, then $f$ is differentiable at $t$ with

$$
f^{\Delta}(t)=\frac{f(\sigma(t))-f(t)}{\mu(t)}=\frac{f(\sigma(t))-f(t)}{\sigma(t)-t}
$$

(iii) If $f$ is right-dense, then $f$ is differentiable at $t$ if and only one the limit

$$
\lim _{s \rightarrow t}=\frac{f(t)-f(s)}{t-s}
$$

exists as a finite number. In this case

$$
f^{\Delta}(t)=\lim _{s \rightarrow t} \frac{f(t)-f(s)}{t-s}
$$

(iv) If $f$ is differentiable at $t$, then

$$
f(\sigma(t))=f(t)+\mu(t) f^{\Delta}(t)=f(t)+(\sigma(t)-t) f^{\Delta}(t) .
$$

Lemma 1.5 (see [7]). Suppose $f: \mathbb{T} \rightarrow \mathbb{R}$ is differentiable at $t_{0} \in \mathbb{T} \backslash\{\max \mathbb{T}\}$. If $f$ assumes its local right-minimum at $t_{0}$, then $f^{\Delta}\left(t_{0}\right) \geq 0$. If $f$ assumes its local right-maximum at $t_{0}$, then $f^{\Delta}\left(t_{0}\right) \leq 0$. 
Lemma 1.6 ((Mean Value Theorem) [7]). Let $f$ be a continuous function on $[a, b]$ that is differentiable on $[a, b)$. Then there exist $\xi^{*}, \tau^{*} \in[a, b)$ such that

$$
f^{\Delta}\left(\tau^{*}\right) \leq \frac{f(b)-f(a)}{b-a} \leq f^{\Delta}\left(\xi^{*}\right)
$$

Lemma 1.7 (see [3]). Suppose $f$ and $g$ are pre-differential with $D$. If $U$ is a compact interval with endpoints $r, s \in \mathbb{T}$, then $|f(s)-f(r)| \leq\left\{\sup _{t \in U^{\kappa} \cap D \cap \mathbb{T}}\left|f^{\Delta}(t)\right|\right\}|s-r|$.

Now, we can obtain the following lemma which is similar to Lemma 1.7. The proofs are similar to the proofs of Lemma 1.7 by a slight modification and we omit the proofs.

Lemma 1.8. Suppose $f(t, u)$ and $g(t, u)$ are predifferential with $D \times(0,+\infty)$. If $U$ is a compact interval with endpoints $r, s \in \mathbb{T}$, then $|f(s, u)-f(r, u)| \leq\left\{\sup _{t \in U^{\star} \cap D \cap \mathbb{T}}\left|f^{\Delta}(t, u)\right|\right\}|s-r|$, here $D \subset \mathbb{T}^{\kappa}$.

Throughout this paper, it is assumed that

(H1) $f(t, u):(0, T)_{\mathbb{T}} \times(0, \infty) \rightarrow \mathbb{R}$ is continuous;

(H2) $q \in C\left((0, T)_{\mathbb{T}},(0, \infty)\right)$ and $q \in C_{l d}^{\nabla}[0, T]_{\mathbb{T}}$;

(H3) $\psi_{i}: \mathbb{R} \rightarrow \mathbb{R}$ are continuous and nondecreasing, here $i=1,2, \ldots, m$.

\section{Existence Results}

Define the Banach space $\mathbb{B}=C[0, T]_{\mathbb{T}}$ with the norm $\|y\|=\sup _{t \in[0, T]_{T}}|y(t)|$.

To demonstrate existence of positive solutions to problem (1.1) and (1.2), we first approximate the singular problem by means of a sequence of nonsingular problems, and by using the lower and upper solution for nonsingular problem together with Schauders fixed point theorem, and then we establish the existence of solutions to each approximating problem. Our results are new even for the corresponding differential $(\mathbb{T}=\mathbb{R})$ and difference equations $(\mathbb{T}=\mathbb{Z})$, as well as in general time scales setting. If we consider the corresponding differential equation $(\mathbb{T}=\mathbb{R})$ of problem (1.1) and (1.2) in the method mentioned above, we obtain the same existence results to problem (1.1) and (1.2). In the same way, we consider the corresponding difference equation $(\mathbb{T}=\mathbb{R})$ of problem $(1.1)$ and $(1.2)$, we obtain the same existence results to problem (1.1) and (1.2). Here, the two same existence results are obtained in different settings by using the essentially same method. Naturally, it is quite necessary to consider the existence results to problem (1.1) and (1.2) in same setting. In this case, we need to solve the problem with the help of calculus on time scales, because it not only can unify differential and difference equations, but also can provide accurate information of phenomena that manifests themselves partly in continuous time and partly in discrete time. For example, we can consider the problem (1.1) and (1.2) on time scales

$$
\mathbb{T}=\{0\} \cup\left\{\left(\frac{1}{2}\right)^{\mathbb{N}}\right\} \cup\left[\frac{1}{2}, 1\right] \cup[2,3] .
$$

However, if $t$ is taken from (2.1), we cannot study the problem (1.1) and (1.2) only in differential case, neither can we study the problem (1.1) and (1.2) only in difference case.

Now we state and prove our main result. 
Theorem 2.1. Let $n_{0} \in\{1,2, \ldots\}$ be fixed. Assume that (H1)-(H3) hold and the following conditions are satisfied.

(A1) For each $n \in\left\{n_{0}, n_{0}+1, \ldots\right\} \equiv \mathbb{N}_{1}$, there is a constant $\rho_{n}$ such that $\left\{\rho_{n}\right\}$ is a strictly monotone decreasing sequence with $\lim _{n \rightarrow \infty} \rho_{n}=0$, and $q(t) f\left(t, \rho_{n}\right) \geq 0$ for $t \in\left[1 / 2^{n+1}, T\right]_{\mathbb{T}} ;$

(A2) There exists a function $\alpha \in C[0, T]_{\mathbb{T}} \cap C^{\Delta}(0, T]_{\mathbb{T}}, \varphi_{p}\left(\alpha^{\Delta}\right) \in C^{\nabla}(0, T)_{\mathbb{T}}$ with $\alpha(0)=$ $0, \alpha(T)-\sum_{i=1}^{m} \psi_{i}\left(\alpha^{\Delta}\left(\xi_{i}\right)\right) \leq 0, \alpha>0$ on $(0, T]_{\mathbb{T}}$ and $-\left(\varphi_{p}\left(\alpha^{\Delta}\right)\right)^{\nabla} \leq q(t) f(t, \alpha)$ for $t \in$ $(0, T)_{\mathbb{T}} ;$

(A3) There exists a function $\beta \in C[0, T]_{\mathbb{T}} \cap C^{\Delta}(0, T]_{\mathbb{T}}, \varphi_{p}\left(\beta^{\Delta}\right) \in C^{\nabla}(0, T)_{\mathbb{T}}$ with $\beta \geq \alpha, \beta \geq$ $\rho_{n_{0}}$ for $t \in[0, T]_{\mathbb{T}}$ and $\beta(T)-\sum_{i=1}^{m} \psi_{i}\left(\beta^{\Delta}\left(\xi_{i}\right)\right)>0$, with $-\left(\varphi_{p}\left(\beta^{\Delta}\right)\right)^{\nabla} \geq q(t) f(t, \beta)$ for $t \in(0, T)_{\mathbb{T}}$, and $-\left(\varphi_{p}\left(\beta^{\Delta}\right)\right)^{\nabla} \geq q(t) f\left(1 / 2^{n_{0}+1}, \beta\right)$ for $t \in\left(0,1 / 2^{n_{0}+1}\right)_{\mathbb{T}}$.

Then the boundary value problem (1.1) and (1.2) has a positive solution $u \in C[0, T]_{\mathbb{T}} \cap$ $C^{\Delta}(0, T]_{\mathbb{T}}, \varphi_{p}\left(u^{\Delta}\right) \in C^{\nabla}(0, T)_{\mathbb{T}}$ with $u \geq \alpha$ for $t \in[0, T]_{\mathbb{T}}$.

Proof. It follows from the condition (A1) that $1 / 2^{n+1} \in(0, T]_{\mathbb{T}}$ for each $n \in \mathbb{N}_{1}$. That is, $(0, T]_{\mathbb{T}}$ is not empty. Without loss of generality, fix $n \in \mathbb{N}_{1}$. If $\xi_{1}>0$, then we can suppose that $\min _{t \in\left[\xi_{1}, T\right]_{\mathbb{T}}} \alpha(t) \geq \rho_{n}$, let $t_{n} \in\left(0, \xi_{1}\right)_{\mathbb{T}}$ be such that

$$
\alpha\left(t_{n}\right)=\rho_{n}, \quad \alpha \leq \rho_{n} \quad \text { for } t \in\left[0, t_{n}\right]_{\mathbb{T}} .
$$

If $\xi_{1}=0$, then we can suppose that $\min _{t \in\left[\xi_{2}, T\right]_{\mathbb{T}}} \alpha(t) \geq \rho_{n}$, let $t_{n} \in\left(0, \xi_{2}\right)_{\mathbb{T}}$ be such that $(2.2)$ holds. Define

$$
\alpha_{n}(t)=\left\{\begin{array}{ll}
\rho_{n} & \text { if } t \in\left[0, t_{n}\right]_{\mathbb{T}}, \\
\alpha & \text { if } t \in\left[t_{n}, T\right]_{\mathbb{T}},
\end{array} \quad \text { here } \alpha\left(t_{n}\right)=\rho_{n} .\right.
$$

We denote $e_{n}=\left[1 / 2^{n+1}, T\right]_{\mathbb{T}}, \omega_{n}(t)=\max \left\{1 / 2^{n+1}, t\right\}$ for $t \in[0, T]_{\mathbb{T}}$ and

$$
f_{n}(t, x)=\max \left\{f(t, x), f\left(\omega_{n}(t), x\right)\right\}
$$

Define a sequence $h_{n_{0}}(t, x)=f_{n_{0}}(t, x)$ and

$$
h_{n}(t, x)=\min \left\{f_{n_{0}}(t, x), \ldots, f_{n}(t, x)\right\}, \quad n=n_{0}+1, n_{0}+2, \ldots
$$

Then

$$
\begin{gathered}
f(t, x) \leq \cdots \leq h_{n+1}(t, x) \leq h_{n}(t, x) \leq \cdots \leq h_{n_{0}}(t, x) \quad \text { for }(t, x) \in(0, T)_{\mathbb{T}} \times(0, \infty), \\
h_{n}(t, x)=f(t, x) \quad \text { for }(t, x) \in e_{n} \times(0, \infty) .
\end{gathered}
$$


6

Abstract and Applied Analysis

Consider the $p$-Laplacian boundary value problem

$$
\begin{gathered}
\left(\varphi_{p}\left(u^{\Delta}(t)\right)\right)^{\nabla}+q(t) h_{n_{0}}^{*}(t, u(t))=0, \quad t \in(0, T)_{\mathbb{T}}, \\
u(0)=\rho_{n_{0}}, \quad u(T)-\sum_{i=1}^{m} \psi_{i}^{*}\left(u^{\Delta}\left(\xi_{i}\right)\right)=\rho_{n_{0}},
\end{gathered}
$$

where

$$
\begin{aligned}
& h_{n_{0}}^{*}(t, u(t))= \begin{cases}h_{n_{0}}\left(t, \alpha_{n_{0}}(t)\right)+r\left(\alpha_{n_{0}}(t)-u(t)\right), & u(t) \leq \alpha_{n_{0}}(t), \\
h_{n_{0}}(t, u(t)), & \alpha_{n_{0}}(t) \leq u(t) \leq \beta, \\
h_{n_{0}}(t, \beta(t))+r(\beta(t)-u(t)), & u(t) \geq \beta,\end{cases} \\
& \psi_{i}^{*}\left(z_{i}\right)= \begin{cases}\psi_{i}\left(\alpha^{\Delta}\left(\xi_{i}\right)\right), & z_{i} \leq \alpha_{n_{0}}^{\Delta}\left(\xi_{i}\right)=\alpha^{\Delta}\left(\xi_{i}\right), \\
\psi_{i}\left(z_{i}\right), & \alpha_{n_{0}}^{\Delta}\left(\xi_{i}\right) \leq z_{i} \leq \beta^{\Delta}\left(\xi_{i}\right), \quad i=1, \ldots, m, \\
\psi_{i}\left(\beta^{\Delta}\left(\xi_{i}\right)\right), & z_{i} \geq \beta^{\Delta}\left(\xi_{i}\right),\end{cases}
\end{aligned}
$$

and $r: \mathbb{R} \rightarrow[-1,1]$ is the radial retraction function defined by

$$
r(u)= \begin{cases}u, & |u| \leq 1 \\ \frac{u}{|u|}, & |u|>1 .\end{cases}
$$

Suppose

$$
C_{0}[0, T]_{\mathbb{T}}=\left\{u \in C[0, T]_{\mathbb{T}}: u(0)=0\right\} \quad C_{\rho_{n_{0}}}^{\Delta}[0, T]_{\mathbb{T}}=\left\{u \in C^{\Delta}[0, T]_{\mathbb{T}}: u(0)=\rho_{n_{0}}\right\} .
$$

We define the mappings $L_{p} ; F: C_{\rho_{n_{0}}}^{\Delta}[0, T]_{\mathbb{T}} \rightarrow C_{0}[0, T]_{\mathbb{T}} \times \mathbb{R}$ be such that

$$
\begin{gathered}
L_{p} u(t)=\left(\varphi_{p}\left(u^{\Delta}(t)\right)-\varphi_{p}\left(u^{\Delta}(0)\right), u(T)\right), \\
F u(t)=\left(-\int_{0}^{t} q(x) h_{n_{0}}^{*}(x, u(x)) \nabla x, \sum_{i=1}^{m} \psi_{i}^{*}\left(u^{\Delta}\left(\xi_{i}\right)\right)+\rho_{n_{0}}\right) .
\end{gathered}
$$

By using the Arzela-Ascoli theorem on time scales [2], we can show that $F$ is continuous and compact. By using the (2.7), (2.8), (2.13) and (2.14), we obtain

$$
\left(\varphi_{p}\left(u^{\Delta}(t)\right)-\varphi_{p}\left(u^{\Delta}(0)\right), u(T)\right)=\left(-\int_{0}^{t} q(x) h_{n_{0}}^{*}(x, u(x)) \nabla x, \sum_{i=1}^{m} \psi_{i}^{*}\left(u^{\Delta}\left(\xi_{i}\right)\right)+\rho_{n_{0}}\right),
$$


that is

$$
L_{p} u(t)=F u(t)
$$

If

$$
L_{p} v=(u, \gamma) \quad \text { for } u \in C_{0}[0, T]_{\mathbb{T}}, \quad \gamma=\rho_{n_{0}}+\int_{0}^{T} \varphi_{q}(u(x)-u(T)) \Delta x
$$

then $v(t)=\rho_{n_{0}}+\int_{0}^{t} \varphi_{q}(u(x)-u(T)) \Delta x$, hence $L_{p}^{-1}$ exists and is continuous. So

$$
u(t)=L_{p}^{-1} F u(t)
$$

It is clear that solving the boundary value problem (2.7) and (2.8) is equivalent to finding a fixed point of $u=L_{p}^{-1} F u \equiv N u$, where $N=L_{p}^{-1} F: C_{\rho_{n_{0}}}^{\Delta}[0, T]_{\mathbb{T}} \rightarrow C_{\rho_{n_{0}}}^{\Delta}[0, T]_{\mathbb{T}}$ is compact. Schauder's fixed point theorem guarantees that the boundary value problem (2.7) and (2.8) has a solution $u_{n_{0}}(t) \in C^{\Delta}[0, T]_{\mathbb{T}}$ with $\varphi_{p}\left(u_{n_{0}}^{\Delta}(t)\right) \in C^{\nabla}(0, T)_{\mathbb{T}}$.

We first show that

$$
\alpha_{n_{0}}(t) \leq u_{n_{0}}(t) \quad \text { for } t \in[0, T]_{\mathbb{T}}
$$

If (2.19) is not true, the function $u_{n_{0}}(t)-\alpha_{n_{0}}(t)$ has a negative minimum for some $\tau \in(0, T]_{\mathbb{T}}$. We consider two cases, namely, $\tau \in(0, T)_{\mathbb{T}}$ and $\tau=T$.

Case 1. Assume that $\tau \in(0, T)_{\mathbb{T}}$, then we claim

$$
\left(\varphi_{p}\left(u_{n_{0}}^{\Delta}\right)\right)^{\nabla}(\tau) \geq\left(\varphi_{p}\left(\alpha_{n_{0}}^{\Delta}\right)\right)^{\nabla}(\tau)
$$

Since $u_{n_{0}}(t)-\alpha_{n_{0}}(t)$ has a negative minimum for some $\tau \in(0, T)_{\mathbb{T}}$, in view of Definition 1.2, Lemmas 1.4 and 1.5, we have $u_{n_{0}}^{\Delta}(\tau)-\alpha_{n_{0}}^{\Delta}(\tau) \geq 0$ and there exists a $\delta$ with $\tau-\delta \in[0, \tau)_{\mathbb{T}}$ such that $u_{n_{0}}^{\Delta}(t)-\alpha_{n_{0}}^{\Delta}(t) \leq 0$ for $t \in[\tau-\delta, \tau)_{\mathbb{T}}$. Thus

$$
\varphi_{p}\left(u_{n_{0}}^{\Delta}(t)\right)-\varphi_{p}\left(\alpha_{n_{0}}^{\Delta}(t)\right) \leq \varphi_{p}\left(u_{n_{0}}^{\Delta}(\tau)\right)-\varphi_{p}\left(\alpha_{n_{0}}^{\Delta}(\tau)\right) \text { for } t \in[\tau-\delta, \tau)_{\mathbb{T}}
$$

which leads to

$$
\frac{\varphi_{p}\left(u_{n_{0}}^{\Delta}(t)\right)-\varphi_{p}\left(u_{n_{0}}^{\Delta}(\tau)\right)}{t-\tau} \geq \frac{\varphi_{p}\left(\alpha_{n_{0}}^{\Delta}(t)\right)-\varphi_{p}\left(\alpha_{n_{0}}^{\Delta}(\tau)\right)}{t-\tau} \quad \text { for } t \in[\tau-\delta, \tau)_{\mathbb{T}}
$$


If $\tau$ is left-dense, in view of Lemma 1.4

$$
\begin{aligned}
\left(\varphi_{p}\left(u_{n_{0}}^{\Delta}\right)\right)^{\nabla}(\tau) & =\lim _{t \in[\tau-\delta, \tau) \rightarrow \tau} \frac{\varphi_{p}\left(u_{n_{0}}^{\Delta}(t)\right)-\varphi_{p}\left(u_{n_{0}}^{\Delta}(\tau)\right)}{t-\tau} \\
& \geq \lim _{t \in[\tau-\delta, \tau) \rightarrow \tau} \frac{\varphi_{p}\left(\alpha_{n_{0}}^{\Delta}(t)\right)-\varphi_{p}\left(\alpha_{n_{0}}^{\Delta}(\tau)\right)}{t-\tau} \\
& =\left(\varphi_{p}\left(\alpha_{n_{0}}^{\Delta}\right)\right)^{\nabla}(\tau) .
\end{aligned}
$$

If $\tau$ is left-scattered, by Lemma 1.4 and (2.22) we obtain

$$
\begin{aligned}
\left(\varphi_{p}\left(u_{n_{0}}^{\Delta}\right)\right)^{\nabla}(\tau) & =\frac{\varphi_{p}\left(u_{n_{0}}^{\Delta}(\tau)\right)-\varphi_{p}\left(u_{n_{0}}^{\Delta}(\rho(\tau))\right)}{\tau-\rho(\tau)} \\
& \geq \frac{\varphi_{p}\left(\alpha_{n_{0}}^{\Delta}(\tau)\right)-\varphi_{p}\left(\alpha_{n_{0}}^{\Delta}(\rho(\tau))\right)}{\tau-\rho(\tau)} \\
& =\left(\varphi_{p}\left(\alpha_{n_{0}}^{\Delta}\right)\right)^{\nabla}(\tau) .
\end{aligned}
$$

Hence, (2.20) is established.

However, by (2.3), (2.9) and $u_{n_{0}}(\tau)<\alpha_{n_{0}}(\tau)$, we obtain

$$
\begin{aligned}
& \left(\varphi_{p}\left(u_{n_{0}}^{\Delta}(\tau)\right)\right)^{\nabla}-\left(\varphi_{p}\left(\alpha_{n_{0}}^{\Delta}(\tau)\right)\right)^{\nabla} \\
& \quad=-\left[q(\tau) h_{n_{0}}\left(\tau, \alpha_{n_{0}}(\tau)\right)+q(\tau) r\left(\alpha_{n_{0}}(\tau)-u_{n_{0}}(\tau)\right)+\left(\varphi_{p}\left(\alpha_{n_{0}}^{\Delta}(\tau)\right)\right)^{\nabla}\right] \\
& \quad= \begin{cases}-\left[q(\tau) h_{n_{0}}(\tau, \alpha(\tau))+q(\tau) r\left(\alpha(\tau)-u_{n_{0}}(\tau)\right)+\left(\varphi_{p}\left(\alpha^{\Delta}(\tau)\right)\right)^{\nabla}\right], & \tau \in\left[t_{n_{0}}, T\right)_{\mathbb{T}}, \\
-\left[q(\tau) h_{n_{0}}\left(\tau, \rho_{n_{0}}\right)+q(\tau) r\left(\rho_{n_{0}}-u_{n_{0}}(\tau)\right)\right], & \tau \in\left(0, t_{n_{0}}\right)_{\mathbb{T}} .\end{cases}
\end{aligned}
$$
we have

Assume that $\tau \in\left[1 / 2^{n_{0}+1}, T\right]_{\mathbb{T}}$, then $g_{n_{0}}(\tau, x)=f(\tau, x)$ for $x \in(0, \infty)$, by (A1) and (A2),

$$
\begin{aligned}
& \left(\varphi_{p}\left(u_{n_{0}}^{\Delta}(\tau)\right)\right)^{\nabla}-\left(\varphi_{p}\left(\alpha_{n_{0}}^{\Delta}(\tau)\right)\right)^{\nabla} \\
& \quad= \begin{cases}-\left[q(\tau) f(\tau, \alpha(\tau))+q(\tau) r\left(\alpha(\tau)-u_{n_{0}}(\tau)\right)+\left(\varphi_{p}\left(\alpha^{\Delta}(\tau)\right)\right)^{\nabla}\right], & \tau \in\left[t_{n_{0}}, T\right)_{\mathbb{T}}, \\
-\left[q(\tau) f\left(\tau, \rho_{n_{0}}\right)+q(\tau) r\left(\rho_{n_{0}}-u_{n_{0}}(\tau)\right)\right], & \tau \in\left(0, t_{n_{0}}\right)_{\mathbb{T}},\end{cases} \\
& \quad<0,
\end{aligned}
$$

which implies a contraction. 
Assume that $\tau \in\left(0,1 / 2^{n_{0}+1}\right)_{\mathbb{T}}$, then $h_{n_{0}}(\tau, x)=\max \left\{f\left(1 / 2^{n_{0}+1}, x\right), f(\tau, x)\right\}$, in view of (A1), (A2) and $q(\tau)>0$, we have

$$
\begin{aligned}
& \left(\varphi_{p}\left(u_{n_{0}}^{\Delta}(\tau)\right)\right)^{\nabla}-\left(\varphi_{p}\left(\alpha_{n_{0}}^{\Delta}(\tau)\right)\right)^{\nabla} \\
& \quad \leq \begin{cases}-\left[q(\tau) f(\tau, \alpha(\tau))+q(\tau) r\left(\alpha(\tau)-u_{n_{0}}(\tau)\right)+\left(\varphi_{p}\left(\alpha^{\Delta}(\tau)\right)\right)^{\nabla}\right], & \tau \in\left[t_{n_{0}}, T\right)_{\mathbb{T}}, \\
-\left[q(\tau) f\left(\frac{1}{2^{n_{0}+1}}, \rho_{n_{0}}\right)+q(\tau) r\left(\rho_{n_{0}}-u_{n_{0}}(\tau)\right)\right], & \tau \in\left(0, t_{n_{0}}\right)_{\mathbb{T}},\end{cases} \\
& \quad<0 .
\end{aligned}
$$

which implies a contraction.

Case 2. Assume that $\tau=T$. That is, $\alpha_{n_{0}}(T)-u_{n_{0}}(T)>0$, by (2.3), (2.8) and (2.10) together with $\alpha(T) \leq \sum_{i=1}^{m} \psi_{i}\left(\alpha^{\Delta}\left(\xi_{i}\right)\right)$, we have the following three subcases.

(a) If $u_{n_{0}}^{\Delta}\left(\xi_{i}\right) \leq \alpha^{\Delta}\left(\xi_{i}\right)$ for $i=1,2, \ldots, m$, then

$$
\begin{aligned}
0 & <\alpha_{n_{0}}(T)-u_{n_{0}}(T) \\
& =\alpha(T)-\sum_{i=1}^{m} \psi_{i}^{*}\left(u_{n_{0}}^{\Delta}\left(\xi_{i}\right)\right)-\rho_{n_{0}} \\
& <\sum_{i=1}^{m} \psi_{i}\left(\alpha^{\Delta}\left(\xi_{i}\right)\right)-\sum_{i=1}^{m} \psi_{i}^{*}\left(u_{n_{0}}^{\Delta}\left(\xi_{i}\right)\right) \\
& =\sum_{i=1}^{m} \psi_{i}\left(\alpha^{\Delta}\left(\xi_{i}\right)\right)-\sum_{i=1}^{m} \psi_{i}\left(\alpha^{\Delta}\left(\xi_{i}\right)\right)=0,
\end{aligned}
$$

this is a contradiction.

(b) If $\alpha^{\Delta}\left(\xi_{i}\right)<u_{n_{0}}^{\Delta}\left(\xi_{i}\right)$ for $i=1,2, \ldots, m$. Assume that $u_{n_{0}}^{\Delta}\left(\xi_{i}\right) \leq \beta^{\Delta}\left(\xi_{i}\right)$ for $i=1,2, \ldots, m$, then

$$
\sum_{i=1}^{m} \psi_{i}^{*}\left(u_{n_{0}}^{\Delta}\left(\xi_{i}\right)\right)=\sum_{i=1}^{m} \psi_{i}\left(u_{n_{0}}^{\Delta}\left(\xi_{i}\right)\right)
$$

Assume that $\beta^{\Delta}\left(\xi_{i}\right)<u_{n_{0}}^{\Delta}\left(\xi_{i}\right)$ for $i=1,2, \ldots, m$, then

$$
\sum_{i=1}^{m} \psi_{i}^{*}\left(u_{n_{0}}^{\Delta}\left(\xi_{i}\right)\right)=\sum_{i=1}^{m} \psi_{i}\left(\beta^{\Delta}\left(\xi_{i}\right)\right)
$$

Assume that there exist sequences $\left\{i_{l_{1}}\right\}=\left\{1,2, \ldots, l_{1}\right\}$ and $\left\{i_{k_{1}}\right\}=\left\{1,2, \ldots, k_{1}\right\}$ such that $\beta^{\Delta}\left(\xi_{i_{1}}\right)<u_{n_{0}}^{\Delta}\left(\xi_{i_{l}}\right)$ and $u_{n_{0}}^{\Delta}\left(\xi_{i_{k_{1}}}\right) \leq \beta^{\Delta}\left(\xi_{i_{1}}\right)$, here $l_{1}+k_{1}=m$, then

$$
\sum_{i=1}^{m} \psi_{i}^{*}\left(u_{n_{0}}^{\Delta}\left(\xi_{i}\right)\right)=\sum_{i_{k_{1}}=1}^{k_{1}} \psi_{i_{k_{1}}}\left(u_{n_{0}}^{\Delta}\left(\xi_{i_{k_{1}}}\right)\right)+\sum_{i_{l_{1}}=1}^{l_{1}} \psi_{i_{l_{1}}}\left(\beta^{\Delta}\left(\xi_{i_{l_{1}}}\right)\right) .
$$


Hence, by (2.29), (2.30) and (2.31) together with the monotonicity of $\psi_{i}$, we have

$$
\begin{aligned}
0 & <\alpha_{n_{0}}(T)-u_{n_{0}}(T) \\
& =\alpha(T)-\sum_{i=1}^{m} \psi_{i}^{*}\left(u_{n_{0}}^{\Delta}\left(\xi_{i}\right)\right)-\rho_{n_{0}} \\
& \leq \sum_{i=1}^{m} \psi_{i}\left(\alpha^{\Delta}\left(\xi_{i}\right)\right)-\sum_{i=1}^{m} \psi_{i}^{*}\left(u_{n_{0}}^{\Delta}\left(\xi_{i}\right)\right)-\rho_{n_{0}}<0,
\end{aligned}
$$

this is a contradiction.

(c) If there exist sequences $\left\{i_{l}\right\}=\{1,2, \ldots, l\}$ and $\left\{i_{k}\right\}=\{1,2, \ldots, k\}$ such that $\alpha^{\Delta}\left(\xi_{i_{l}}\right)<$ $u_{n_{0}}^{\Delta}\left(\xi_{i_{l}}\right)$ and $u_{n_{0}}^{\Delta}\left(\xi_{i_{k}}\right) \leq \alpha^{\Delta}\left(\xi_{i_{k}}\right)$, here $l+k=m$. Essentially the same reasoning as before we have $0<\alpha_{n_{0}}(T)-u_{n_{0}}(T)=\alpha(T)-\sum_{i=1}^{m} \psi_{i}^{*}\left(u_{n_{0}}^{\Delta}\left(\xi_{i}\right)\right)-\rho_{n_{0}}<0$, this is a contradiction.

Thus, Cases 1-2 imply (2.19) is established. In particular, since $\alpha(t) \leq \alpha_{n_{0}}(t)$ for $t \in$ $[0, T]_{\mathbb{T}}$, we obtain $\alpha(t) \leq \alpha_{n_{0}}(t) \leq u_{n_{0}}(t)$ for $t \in[0, T]_{\mathbb{T}}$.

Essentially the same reasoning as the proof of inequality (2.19) we obtain $u_{n_{0}}(t) \leq$ $\beta$ for $t \in[0, T]_{\mathbb{T}}$.

Hence

$$
\alpha(t) \leq \alpha_{n_{0}}(t) \leq u_{n_{0}}(t) \leq \beta(t) \quad \text { for } t \in[0, T]_{\mathbb{T}} .
$$

Now, we discuss the boundary value problem

$$
\begin{gathered}
\left(\varphi_{p}\left(u^{\Delta}(t)\right)\right)^{\nabla}+q(t) h_{n_{0}+1}^{*}(t, u(t))=0, \quad t \in(0, T)_{\mathbb{T}} \\
u(0)=\rho_{n_{0}+1}, \quad u(T)-\sum_{i=1}^{m} \psi_{i}^{*}\left(u^{\Delta}\left(\xi_{i}\right)\right)=\rho_{n_{0}+1}
\end{gathered}
$$

where

$$
\begin{aligned}
& h_{n_{0}+1}^{*}(t, u(t))= \begin{cases}h_{n_{0}+1}\left(t, \alpha_{n_{0}+1}(t)\right)+r\left(\alpha_{n_{0}+1}(t)-u(t)\right), & u(t) \leq \alpha_{n_{0}+1}(t), \\
h_{n_{0}+1}(t, u(t)), & \alpha_{n_{0}+1}(t) \leq u(t) \leq u_{n_{0}}(t), \\
h_{n_{0}+1}\left(t, u_{n_{0}}(t)\right)+r\left(u_{n_{0}}(t)-u(t)\right), & u(t) \geq u_{n_{0}}(t),\end{cases} \\
& \psi_{i}^{*}\left(z_{i}\right)= \begin{cases}\psi_{i}\left(\alpha^{\Delta}\left(\xi_{i}\right)\right), & z_{i} \leq \alpha_{n_{0}+1}^{\Delta}\left(\xi_{i}\right)=\alpha^{\Delta}\left(\xi_{i}\right) \\
\psi_{i}\left(z_{i}\right), & \alpha_{n_{0}+1}^{\Delta}\left(\xi_{i}\right) \leq z_{i} \leq u_{n_{0}}^{\Delta}\left(\xi_{i}\right), \quad i=1, \ldots, m . \\
\psi_{i}\left(u_{n_{0}}^{\Delta}\left(\xi_{i}\right)\right), & z_{i} \geq u_{n_{0}}^{\Delta}\left(\xi_{i}\right)\end{cases}
\end{aligned}
$$

Schauder's fixed point theorem guarantees that the boundary value problem (2.34) has a solution $u_{n_{0}+1}(t) \in C^{\Delta}[0, T]_{\mathbb{T}}$ with $\varphi_{p}\left(u_{n_{0}+1}^{\Delta}(t)\right) \in C^{\nabla}(0, T)_{\mathbb{T}}$. 
Essentially the same reasoning as the proof of inequality (2.33), we have

$$
\alpha(t) \leq \alpha_{n_{0}+1}(t) \leq u_{n_{0}+1}(t) \leq u_{n_{0}}(t) \text { for } t \in[0, T]_{\mathbb{T}} .
$$

If there exists $u_{k}(t)$ for some $k \in\left\{n_{0}+1, n_{0}+2, \ldots\right\}$ satisfying $\alpha_{k}(t) \leq u_{k}(t) \leq u_{k-1}(t)$ for $t \in[0, T]_{\mathbb{T}}$. Then we investigate the boundary value problem

$$
\begin{gathered}
\left(\varphi_{p}\left(u^{\Delta}(t)\right)\right)^{\nabla}+q(t) h_{k+1}^{*}(t, u(t))=0, \quad t \in(0, T)_{\mathbb{T}}, \\
u(0)=\rho_{k+1}, \quad u(T)-\sum_{i=1}^{m} \psi_{i}^{*}\left(u^{\Delta}\left(\xi_{i}\right)\right)=\rho_{k+1},
\end{gathered}
$$

where

$$
\begin{aligned}
& h_{k+1}^{*}(t, u(t))= \begin{cases}h_{k+1}\left(t, \alpha_{k+1}(t)\right)+r\left(\alpha_{k+1}(t)-u(t)\right), & u(t) \leq \alpha_{k+1}(t), \\
h_{k+1}(t, u(t)), & \alpha_{k+1}(t) \leq u(t) \leq u_{k}(t), \\
h_{k+1}\left(t, u_{k}(t)\right)+r\left(u_{k}(t)-u(t)\right), & u(t) \geq u_{k}(t),\end{cases} \\
& \psi_{i}^{*}\left(z_{i}\right)= \begin{cases}\psi_{i}\left(\alpha^{\Delta}\left(\xi_{i}\right)\right), & z_{i} \leq \alpha_{k+1}^{\Delta}\left(\xi_{i}\right)=\alpha^{\Delta}\left(\xi_{i}\right), \\
\psi_{i}\left(z_{i}\right), & \alpha_{k+1}^{\Delta}\left(\xi_{i}\right) \leq z_{i} \leq u_{k}^{\Delta}\left(\xi_{i}\right), \quad i=1, \ldots, m \\
\psi_{i}^{\Delta}\left(u_{k}\left(\xi_{i}\right)\right), & z_{i} \geq u_{k}\left(\xi_{i}\right),\end{cases}
\end{aligned}
$$

It follows from Schauder's fixed point theorem that the boundary value problem (2.37) has a solution $u_{k+1}(t) \in C^{\Delta}[0, T]_{\mathbb{T}}$ with $\varphi_{p}\left(u_{k+1}^{\Delta}(t)\right) \in C^{\nabla}(0, T)_{\mathbb{T}}$.

By using the similar arguments as above, we have

$$
\alpha(t) \leq \alpha_{k+1}(t) \leq u_{k+1}(t) \leq u_{k}(t) \text { for } t \in[0, T]_{\mathbb{T}} .
$$

Hence, for each $n \in\left\{n_{0}, n_{0}+1, \ldots\right\}$, the mathematical induction implies that

$$
\alpha(t) \leq \alpha_{n}(t) \leq u_{n}(t) \leq u_{n-1}(t) \leq \cdots \leq u_{n_{0}}(t) \leq \beta(t) \quad \text { for } t \in[0, T]_{\mathbb{T}} .
$$

Denote

$$
R_{n_{0}}=\sup \left\{|f(t, y)|: t \in\left[\frac{1}{2^{n_{0}+1}}, T\right]_{\mathbb{T}}, \alpha(t) \leq y \leq u_{n_{0}}(t)\right\}
$$

It follows from Lemma 1.6 that there exist $\tau_{1}^{*}, \tau_{2}^{*} \in\left[1 / 2^{n_{0}+1}, T\right)_{\mathbb{T}}$ satisfy

$$
u_{n}^{\Delta}\left(\tau_{1}^{*}\right) \leq \frac{u_{n}(T)-u_{n}\left(1 / 2^{n_{0}+1}\right)}{T-1 / 2^{n_{0}+1}} \leq u_{n}^{\Delta}\left(\tau_{2}^{*}\right)
$$


From (2.42), we have

$$
\begin{aligned}
\varphi_{p}\left(u_{n}^{\Delta}(t)\right) & =\varphi_{p}\left(u_{n}^{\Delta}\left(\tau_{1}^{*}\right)\right)-\int_{\tau_{1}^{*}}^{t} q(s) f(s, u(s)) \nabla s \\
& \leq \varphi_{p}\left(\frac{u_{n}(T)-u_{n}\left(1 / 2^{n_{0}+1}\right)}{T-1 / 2^{n_{0}+1}}\right)+R_{n_{0}} \int_{1 / 2^{n_{0}+1}}^{T} q(s) \nabla s \quad \text { for } t \in\left[\frac{1}{2^{n_{0}+1}}, T\right]_{\mathbb{T}}, \\
\varphi_{p}\left(u_{n}^{\Delta}(t)\right) & =\varphi_{p}\left(u_{n}^{\Delta}\left(\tau_{2}^{*}\right)\right)-\int_{\tau_{2}^{*}}^{t} q(s) f(s, u(s)) \nabla s \\
& \geq \varphi_{p}\left(\frac{u_{n}(T)-u_{n}\left(1 / 2^{n_{0}+1}\right)}{T-1 / 2^{n_{0}+1}}\right)-R_{n_{0}} \int_{1 / 2^{n_{0}+1}}^{T} q(s) \nabla s \text { for } t \in\left[\frac{1}{2^{n_{0}+1}}, T\right]_{\mathbb{T}} .
\end{aligned}
$$

So there exists a positive number $K_{0}$ such that $\left|u_{n}^{\Delta}(t)\right| \leq K_{0}$. By Lemma 1.8, we have

$$
\left\{u_{n}(t)\right\}_{n=n_{0}+1}^{\infty} \text { is a bounded, equicontinuous family on } t \in\left[\frac{1}{2^{n_{0}+1}}, T\right]_{\mathbb{T}} .
$$

The Arzela-Ascoli theorem on time scales [2] guarantees the existence of a subsequence $\mathbb{N}_{n_{0}}$ of integers and a function $z_{n_{0}}(t) \in C\left[1 / 2^{n_{0}+1}, T\right]_{\mathbb{T}}$ with $u_{n}(t)$ converging uniformly to $z_{n_{0}}(t)$ on $\left[1 / 2^{n_{0}+1}, T\right]_{\mathbb{T}}$ as $n \rightarrow \infty$ through $\mathbb{N}_{n_{0}}$. Similarly

$$
\left\{u_{n}(t)\right\}_{n=n_{0}+1}^{\infty} \text { is a bounded, equicontinuous family on } t \in\left[\frac{1}{2^{n_{0}+2}}, T\right]_{\mathbb{T}} .
$$

Thus there is a subsequence $\mathbb{N}_{n_{0}+1}$ of $\mathbb{N}_{n_{0}}$ and a function $z_{n_{0}+1}(t) \in C\left[1 / 2^{n_{0}+2}, T\right]_{\mathbb{T}}$ with $u_{n}(t)$ converging uniformly to $z_{n_{0}+1}(t)$ on $\left[1 / 2^{n_{0}+2}, T\right]_{\mathbb{T}}$ as $n \rightarrow \infty$ through $\mathbb{N}_{n_{0}+1}$. Since $\mathbb{N}_{n_{0}+1} \subseteq \mathbb{N}_{n_{0}}$, we have $z_{n_{0}+1}(t)=z_{n_{0}}(t)$ on $\left[1 / 2^{n_{0}+1}, T\right]_{\mathbb{T}}$. Proceed inductively to obtain subsequence of integers $\mathbb{N}_{n_{0}} \supseteq \mathbb{N}_{n_{0}+1} \supseteq \cdots \supseteq \mathbb{N}_{n} \supseteq \cdots$ and functions $z(t) \in C\left[1 / 2^{n+1}, T\right]_{\mathbb{T}}$ with $u_{n}(t)$ converging uniformly to $z_{n}(t)$ on $\left[1 / 2^{n+1}, T\right]_{\mathbb{T}}$ as $n \rightarrow \infty$ through $\mathbb{N}_{n}$ and $z_{n}(t)=$ $z_{n-1}(t)$ on $\left[1 / 2^{n}, T\right]_{\mathbb{T}}$.

Now, we define a function $u:[0, T] \rightarrow[0, \infty)$ with $u(t)=z_{n}(t)$ on $\left[1 / 2^{n+1}, T\right]_{\mathbb{T}}$ and $u(0)=0$. Notice, $u(t)$ is well defined and $\alpha(t) \leq u(t) \leq u_{n_{0}}(t) \leq \beta$ for $t \in(0, T)_{\mathbb{T}}$. Nextly fix $t \in(0, T)_{\mathbb{T}}$ and let $l \in\left\{n_{0}, n_{0}+1, \ldots\right\}$ be such that $t \in\left(1 / 2^{l+1}, T\right)_{\mathbb{T}}$, let $\mathbb{N}_{l}^{*}=\left\{n \in \mathbb{N}_{l}: n \geq l\right\}$, we have

$$
\psi_{i}^{*}\left(u_{n}\left(\xi_{i}\right)\right)=\psi_{i}\left(u_{n}\left(\xi_{i}\right)\right), \quad h_{n}^{*}\left(t, u_{n}(t)\right)=h_{n}\left(t, u_{n}(t)\right)=f\left(t, u_{n}(t)\right) \quad \text { for } n \in \mathbb{N}_{l}^{*} .
$$


Hence, for $n \in \mathbb{N}_{1}^{*}$, we have $u_{n}$ which is the positive solution of the following boundary value problem

$$
\begin{gathered}
\left(\varphi_{p}\left(u_{n}^{\Delta}(t)\right)\right)^{\nabla}+q(t) f\left(t, u_{n}(t)\right)=0, \quad t \in\left(\frac{1}{2^{n}}, T\right)_{\mathbb{T}}, \\
u_{n}(0)=\rho_{n}, \quad u_{n}(T)-\sum_{i=1}^{m} \psi_{i}\left(u_{n}^{\Delta}\left(\xi_{i}\right)\right)=\rho_{n} .
\end{gathered}
$$

Let $n \rightarrow \infty$ through $\mathbb{N}_{l}^{*}$, we have $u(t)$ that satisfies

$$
\begin{gathered}
\left(\varphi_{p}\left(u^{\Delta}(t)\right)\right)^{\nabla}+q(t) f(t, u(t))=0, \quad t \in(0, T)_{\mathbb{T}}, \\
u(0)=0, \quad u(T)-\sum_{i=1}^{m} \psi_{i}\left(u^{\Delta}\left(\xi_{i}\right)\right)=0 .
\end{gathered}
$$

It remains to show that $u(t)$ is continuous at 0 . Now by $\lim _{n \rightarrow \infty} u_{n}(0)=0$, there exists $n_{1} \in$ $\left\{n_{0}, n_{0}+1, \ldots\right\}$ with $u_{n_{1}}(0)<\varepsilon / 2$. Since $u_{n_{1}}(t) \in C[0, T]_{\mathbb{T}}$ there exists $\delta_{n_{1}} \in(0, T)_{\mathbb{T}}$ with $u_{n_{1}}(t)<\varepsilon / 2$ for $t \in\left[0, \delta_{n_{1}}\right)_{\mathbb{T}}$. By the monotonicity of $\left\{u_{n}(t)\right\}_{n \in \mathbb{N}_{0}}$ for each $t \in[0, T]_{\mathbb{T}}$, we have $\alpha(t) \leq u_{n}(t) \leq u_{n_{1}}(t)<\varepsilon / 2$ for $t \in\left[0, \delta_{n_{1}}\right)_{\mathbb{T}}$ and $n \geq n_{1}$, which means $\alpha(t) \leq u(t)<\varepsilon / 2$ for $t \in\left[0, \delta_{n_{1}}\right)_{\mathbb{T}}$. So $u(t)$ is continuous at 0 .

If we replace $t \in\left[1 / 2^{n+1}, T\right]_{\mathbb{T}}$ with $t \in\left[1 / 2^{n+1}, T-1 / 2^{n+1}\right]_{\mathbb{T}}$, the singularity occurs at $u=0, t=0$ and $t=T$.

If we replace $t \in\left[1 / 2^{n+1}, T\right]_{\mathbb{T}}$ with $t \in\left[0, T-1 / 2^{n+1}\right]_{\mathbb{T}}$, the singularity occurs at $u=0$ and $t=T$.

If we replace $t \in\left[1 / 2^{n+1}, T\right]_{\mathbb{T}}$ with $t \in[0, T]_{\mathbb{T}}$, the singularity occurs at $u=0$.

So it is easily obtain the analogue of Theorem 2.1 in this section. See the following remark.

Remark 2.2. If (A3) is appropriately adjusted, we can replace $t \in\left[1 / 2^{n+1}, T\right]_{\mathbb{T}}$ in (A1) by

$$
\begin{aligned}
& t \in\left[\frac{1}{2^{n+1}}, T-\frac{1}{2^{n+1}}\right]_{\mathbb{T}}, \\
& t \in\left[0, T-\frac{1}{2^{n+1}}\right]_{\mathbb{T}},
\end{aligned}
$$

or

$$
t \in[0, T]_{\mathbb{T}} .
$$

For example, if (2.49) occurs, (A3) is replaced by

$\left(\mathrm{A}^{\prime}\right)$ There exists a function $\beta \in C[0, T]_{\mathbb{T}} \cap C^{\Delta}(0, T]_{\mathbb{T}}, \varphi_{p}\left(\beta^{\Delta}\right) \in C^{\nabla}(0, T)_{\mathbb{T}}$ such that $\beta \geq \alpha$ and $\beta \geq \rho_{n_{0}}$ for $t \in[0, T]_{\mathbb{T}}, \beta(T)-\sum_{i=1}^{m} \psi_{i}\left(\beta^{\Delta}\left(\xi_{i}\right)\right)>0,-\left(\varphi_{p}\left(\beta^{\Delta}\right)\right)^{\nabla} \geq q(t) f(t, \beta)$ for $t \in$ $(0, T)_{\mathbb{T}},-\left(\varphi_{p}\left(\beta^{\Delta}\right)\right)^{\nabla} \geq q(t) f\left(1 / 2^{n_{0}+1}, \beta\right)$ for $t \in\left(0,1 / 2^{n_{0}+1}\right)_{\mathbb{T}}$ and $-\left(\varphi_{p}\left(\beta^{\Delta}\right)\right)^{\nabla} \geq q(t) f(T-$ $\left.1 / 2^{n_{0}+1}, \beta\right)$ for $t \in\left(T-1 / 2^{n_{0}+1}, T\right)_{\mathbb{T}}$. 
Assume that (H1)-(H3), (A1) and (A2) hold, and in addition suppose the following conditions are satisfied:

(A4) $-\left(\varphi_{p}\left(\alpha^{\Delta}\right)\right)^{\nabla}<q(t) f(t, u)$ for

$$
(t, u) \in(0, T]_{\mathbb{T}} \times\left\{u \in C[0, T]_{\mathbb{T}} \cap C^{\Delta}(0, T]_{\mathbb{T}}: 0<u \leq \alpha\right\} ;
$$

(A5) There exists a function $\beta \in C[0, T]_{\mathbb{T}} \cap C^{\Delta}(0, T]_{\mathbb{T}}, \varphi_{p}\left(\beta^{\Delta}\right) \in C^{\nabla}(0, T)_{\mathbb{T}}$ such that $\beta \geq \rho_{n_{0}}$ for $t \in[0, T]_{\mathbb{T}}, \beta(T)-\sum_{i=1}^{m} \psi_{i}\left(\beta^{\Delta}\left(\xi_{i}\right)\right)>0,-\left(\varphi_{p}\left(\beta^{\Delta}\right)\right)^{\nabla} \geq q(t) f(t, \beta)$ for $t \in(0, T)_{\mathbb{T}}$ and $-\left(\varphi_{p}\left(\beta^{\Delta}\right)\right)^{\nabla} \geq q(t) f\left(1 / 2^{n_{0}+1}, \beta\right)$ for $t \in\left(0,1 / 2^{n_{0}+1}\right)_{\mathbb{T}} ;$

(A6) $\beta(T) \geq \alpha(T)$.

Then the result in Theorem 2.1 is also true. This follows immediately from Theorem 2.1 if we show (A3) holds. That is to say, if we show $\beta \geq \alpha$ for $t \in[0, T]_{\mathbb{T}}$, then the result holds. Assume it is not true, in view of (A6) we obtain $\beta-\alpha$ has a negative minimum for some $\tau_{6} \in(0, T)_{\mathbb{T}}$, so $(\beta-\alpha)^{\Delta}\left(\tau_{6}\right) \geq 0$ and essentially the same reasoning as the proof of inequality (2.20), we have $\left(\varphi_{p}\left(\alpha^{\Delta}\right)\right)^{\nabla}\left(\tau_{6}\right) \leq\left(\varphi_{p}\left(\beta^{\Delta}\right)\right)^{\nabla}\left(\tau_{6}\right)$. However, by (A4), (A5) and $\alpha\left(\tau_{6}\right)>\beta\left(\tau_{6}\right)>0$, we obtain $-\left(\varphi_{p}\left(\alpha^{\Delta}\right)\right)^{\nabla}\left(\tau_{6}\right)<q\left(\tau_{6}\right) f\left(\tau_{6}, \beta\left(\tau_{6}\right)\right)$. Hence $\left(\varphi_{p}\left(\alpha^{\Delta}\right)\right)^{\nabla}\left(\tau_{6}\right)-$ $\left(\varphi_{p}\left(\beta^{\Delta}\right)\right)^{\nabla}\left(\tau_{6}\right) \geq\left(\varphi_{p}\left(\alpha^{\Delta}\right)\right)^{\nabla}\left(\tau_{6}\right)+q\left(\tau_{6}\right) f\left(\tau_{6}, \beta\left(\tau_{6}\right)\right)>0$, which implies a contradiction.

Corollary 2.3. Let $n_{0} \in\{1,2, \ldots\}$ be fixed, suppose (H1)-(H3), (A1), (A2) and (A4)-(A6) hold, then the boundary value problem (1.1) and (1.2) has a solution $u \in C[0, T]_{\mathbb{T}} \cap C^{\Delta}(0, T]_{\mathbb{T}}, \varphi_{p}\left(u^{\Delta}\right) \in$ $C^{\nabla}(0, T)_{\mathbb{T}}$ with $u \geq \alpha$ for $t \in[0, T]_{\mathbb{T}}$.

\section{Construction of $\alpha$ and $\beta$}

In this section, we consider how to construct a lower solution $\alpha$ and an upper solution $\beta$ in certain circumstances. In this section, we assume that

$$
\sum_{i=1}^{m} \psi_{i}\left(x_{i}\right) \geq 0 \quad \text { for } x_{i} \in \mathbb{R}
$$

Lemma 3.1. Assume that there exists a nonincreasing positive sequence $\left\{\varepsilon_{n}\right\}$ with $\lim _{n \rightarrow \infty} \varepsilon_{n}=0$, then there exist a function $\lambda(t) \in C^{\Delta}[0, T]_{\mathbb{T}}$ satisfying

(i) $\varphi_{p}\left(\lambda^{\Delta}(t)\right) \in C^{\nabla}[0, T]_{\mathbb{T}}, \lambda(t)>0$ for $t \in(0, T]_{\mathbb{T}}$ and $\max _{t \in[0, T]_{\mathbb{T}}}\left|\left(\varphi_{p}\left(\lambda^{\Delta}(t)\right)\right)^{\nabla}\right|>0$;

(ii) $\lambda(0)=0, \lambda(T)-\sum_{i=1}^{m} \psi_{i}\left(\lambda^{\Delta}\left(\xi_{i}\right)\right)<0$ and $0<\lambda(t) \leq \varepsilon_{n}$ for $t \in(0, T]_{\mathbb{T}}$.

Proof. Let $e_{n}=\left[1 / 2^{n+1}, T\right]_{\mathbb{T}}\left(n \geq n_{0}\right)$. Assume that $r:[0, T]_{\mathbb{T}} \rightarrow[0, \infty)$ be such that $r(0)=$ $0, r(t)=\varepsilon_{n}^{p-1} /(2 T)^{p+1}$ for $t \in e_{n} \backslash e_{n-1}, n \geq n_{0}$ and $r(t)=\varepsilon_{n_{0}}^{p-1} /(2 T)^{p+1}$ for $t \in\left[1 / 2^{n_{0}}, T\right]_{\mathbb{T}}$. Let $u(t)=\int_{0}^{t} r(s) \Delta s, v(t)=\left[\int_{0}^{t} u(s) \nabla s\right]^{1 /(p-1)}, w(t)=\int_{0}^{t} v(s) \Delta s$. Suppose $\tau_{7} \in e_{n} \backslash e_{n-1}$ for $n \geq$ $n_{0}, \tau_{8} \in(0, T]_{\mathbb{T}}$ satisfy $\tau_{7}<\tau_{8}$ and $2 \tau_{8}-T \geq \tau_{7}$. It is easy to show that $u, v, w:\left[0, \tau_{7}\right]_{\mathbb{T}} \rightarrow[0, \infty)$ are continuous and increasing. Denote

$$
a(t)=\left[c_{0}\left(\tau_{8}-t\right)+c_{1} t\right]^{1 /(p-1)} \quad \text { for } t \in\left[\tau_{7}, T\right]_{\mathbb{T}},
$$


here

$$
c_{0}=-\frac{\tau_{7}}{\tau_{8}} u\left(\tau_{7}\right)+\frac{1}{\tau_{8}}\left(v\left(\tau_{7}\right)\right)^{p-1}, \quad c_{1}=\frac{\tau_{8}-\tau_{7}}{\tau_{8}} u\left(\tau_{7}\right)+\frac{1}{\tau_{8}}\left(v\left(\tau_{7}\right)\right)^{p-1} .
$$

Hence, $a(t)>0$ for $t \in\left[\tau_{7}, T\right]_{\mathbb{T}}$ and is nondecreasing. Define

$$
\begin{gathered}
b(t)=\int_{\tau_{7}}^{t} a(s) \Delta s+w\left(\tau_{7}\right) \quad \text { for } t \in\left[\tau_{7}, \tau_{8}\right]_{\mathbb{T}}, \\
P(t)=\left\{\begin{array}{ll}
b(t), & t \in\left[\tau_{7}, \tau_{8}\right]_{\mathbb{T}}, \\
b\left(2 \tau_{8}-t\right), & t \in\left[\tau_{8}, T\right]_{\mathbb{T}},
\end{array} \quad \lambda(t)= \begin{cases}w(t), & t \in\left[0, \tau_{7}\right]_{\mathbb{T}}, \\
P(t), & t \in\left[\tau_{7}, T\right]_{\mathbb{T}} .\end{cases} \right.
\end{gathered}
$$

We can easily prove $w\left(\tau_{7}\right)=P\left(\tau_{7}\right), w^{\Delta}\left(\tau_{7}\right)=P^{\Delta}\left(\tau_{7}\right),\left(\varphi_{p}\left(w^{\Delta}\right)\right)^{\nabla}\left(\tau_{7}\right)=\left(\varphi_{p}\left(P^{\Delta}\right)\right)^{\nabla}\left(\tau_{7}\right)$ and $w \in C^{\Delta}\left[0, \tau_{7}\right]_{\mathbb{T}}, P \in C^{\Delta}\left[\tau_{7}, T\right]_{\mathbb{T}}, \varphi_{p}\left(w^{\Delta}\right) \in C^{\nabla}\left[0, \tau_{7}\right]_{\mathbb{T}}, \varphi_{p}\left(P^{\Delta}\right) \in C^{\nabla}\left[\tau_{7}, T\right]_{\mathbb{T}}$. Thus, we have $\lambda \in C^{\Delta}[0, T]_{\mathbb{T}}$ and $\varphi_{p}\left(\lambda^{\Delta}\right) \in C^{\nabla}[0, T]_{\mathbb{T}}$ with $\max _{0 \leq t \leq T}\left|\left(\varphi_{p}\left(\lambda^{\Delta}\right)\right)^{\nabla}(t)\right|>0$. Now since $w(t)>0$ for $t \in\left(0, \tau_{7}\right]_{\mathbb{T}}$ and $P(t)>0$ for $t \in\left[\tau_{7}, T\right]_{\mathbb{T}}$, we have $\lambda(t)>0$ for $t \in(0, T]_{\mathbb{T}}$. On the other hand,

$$
\begin{gathered}
u\left(\tau_{7}\right)=\int_{0}^{\tau_{7}} r(s) \Delta s \leq \tau_{7} \frac{\varepsilon_{n}^{p-1}}{(2 T)^{p+1}}<\frac{\varepsilon_{n}^{p-1}}{(2 T)^{p}} \\
v\left(\tau_{7}\right)=\left[\int_{0}^{\tau_{7}} u(s) \nabla s\right]^{1 /(p-1)}<\left(\tau_{7} \frac{\varepsilon_{n}^{p-1}}{(2 T)^{p}}\right)^{1 /(p-1)}<\frac{\varepsilon_{n}}{2^{p /(p-1)} T}, \quad w\left(\tau_{7}\right)<\tau_{7} \times \frac{\varepsilon_{n}}{2^{p /(p-1)} T}<\frac{\varepsilon_{n}}{2},
\end{gathered}
$$

by the monotonicity of $P(t)$ on $\left[\tau_{7}, \tau_{8}\right]_{\mathbb{T}},\left[\tau_{8}, T\right]_{\mathbb{T}}$, respectively, we have

$$
\begin{aligned}
\lambda\left(\tau_{8}\right) & =\max _{t \in\left[\tau_{7}, T\right]_{\mathbb{T}}} \lambda(t) \\
& =\int_{\tau_{7}}^{\tau_{8}} a(s) \Delta s+w\left(\tau_{7}\right) \\
& \leq\left(\tau_{8}-\tau_{7}\right) \max _{t \in\left[\tau_{7}, \tau_{8}\right]_{\mathbb{T}}}\left[c_{0}\left(\tau_{8}-t\right)+c_{1} t\right]^{1 /(p-1)}+w\left(\tau_{7}\right) \\
& \leq\left(\tau_{8}-\tau_{7}\right)\left[\left(\tau_{8}-\tau_{7}\right) u\left(\tau_{7}\right)+\left(v\left(\tau_{7}\right)\right)^{p-1}\right]^{1 /(p-1)}+w\left(\tau_{7}\right) \\
& <T\left[T \frac{\varepsilon_{n}^{p-1}}{(2 T)^{p}}+\frac{\varepsilon_{n}^{p-1}}{2(2 T)^{p-1}}\right]^{1 /(p-1)}+\frac{\varepsilon_{n}}{2}<\frac{\varepsilon_{n}}{2}+\frac{\varepsilon_{n}}{2} \\
& =\varepsilon_{n} .
\end{aligned}
$$

Consequently, $0<\lambda(t) \leq \varepsilon_{n}, t \in(0, T]_{\mathbb{T}}$. 
Without loss of generality, $\sum_{i=1}^{m} \psi_{i}\left(\lambda^{\Delta}\left(\xi_{i}\right)\right) \geq \varepsilon_{n}>\lambda(T)$. We have

$$
\lambda(T)-\sum_{i=1}^{m} \psi_{i}\left(\lambda^{\Delta}\left(\xi_{i}\right)\right)<0
$$

Now we discuss how to construct a lower solution $\alpha(t)$ in (A2) and (A4).

(A7) For each $n \in\{1,2, \ldots\}$, there exist a constant $k_{0}$ and a strictly monotone decreasing sequence $\left\{\rho_{n}\right\}$ with $\lim _{n \rightarrow \infty} \rho_{n}=0$, and $q(t) f(t, u) \geq k_{0}$ for $(t, u) \in\left[1 / 2^{n+1}, T\right]_{\mathbb{T}} \times$ $\left\{u \in C[0, T]_{\mathbb{T}} \cap C^{\Delta}(0, T]_{\mathbb{T}}: 0<u \leq \rho_{n}\right\} ;$

(A8) There exists a function $\beta \in C[0, T]_{\mathbb{T}} \cap C^{\Delta}(0, T]_{\mathbb{T}}, \varphi_{p}\left(\beta^{\Delta}\right) \in C^{\nabla}(0, T)_{\mathbb{T}}$ such that $\beta \geq 0$ for $t \in[0, T]_{\mathbb{T}}, \beta(T)-\sum_{i=1}^{m} \psi_{i}\left(\beta^{\Delta}\left(\xi_{i}\right)\right)>0,-\left(\varphi_{p}\left(\beta^{\Delta}\right)\right)^{\nabla} \geq q(t) f(t, \beta)$ for $t \in(0, T)_{\mathbb{T}}$ and $-\left(\varphi_{p}\left(\beta^{\Delta}\right)\right)^{\nabla} \geq q(t) f\left(1 / 2^{n_{0}+1}, \beta\right)$ for $t \in\left(0,1 / 2^{n_{0}+1}\right)_{\mathbb{T}}$.

Theorem 3.2. Let $n_{0} \in\{3,4, \ldots\}$ be fixed. If (H1)-(H3), (3.1) and (A7)-(A8) hold, then boundary value problem (1.1) and (1.2) has a solution $u \in C[0, T]_{\mathbb{T}} \cap C^{\Delta}(0, T]_{\mathbb{T}}$ with $\varphi_{p}\left(u^{\Delta}\right) \in C^{\nabla}(0, T)_{\mathbb{T}}$ and $u(t)>0$ for $t \in(0, T]_{\mathbb{T}}$.

Proof. By Corollary 2.3, we need only show that conditions (A1), (A2), (A4)-(A6) are satisfied. Without loss of generality, suppose

$$
\beta(t)>\rho_{n_{0}} \quad \text { for } t \in[0, T]_{\mathbb{T}}, \quad \beta(T)-\sum_{i=1}^{m} \psi_{i}\left(\beta^{\Delta}\left(\xi_{i}\right)\right)>\rho_{n_{0}},
$$

by (A7), (A8) and (3.8), we obtain that (A1) and (A5) hold.

From Lemma 3.1 there exists a function $\lambda(t) \in C^{\Delta}[0, T]_{\mathbb{T}}$ satisfying

(i) $\varphi_{p}\left(\lambda^{\Delta}(t)\right) \in C^{\nabla}[0, T]_{\mathbb{T}}, \lambda(t)>0$ for $t \in(0, T]_{\mathbb{T}}$ and $R_{1}=\max _{t \in[0, T]_{\mathbb{T}}}\left|\left(\varphi_{p}\left(\lambda^{\Delta}(t)\right)\right)^{\nabla}\right|>0$.

(ii) $\lambda(0)=0, \lambda(T)-\sum_{i=1}^{m} \psi_{i}\left(\lambda^{\Delta}\left(\xi_{i}\right)\right)<0$ and $0<\lambda(t) \leq \rho_{n}$ for $t \in(0, T]_{\mathbb{T}}$.

Assume $m=\min \left\{1,\left(k_{0} / 2 R_{1}\right)^{1 /(p-1)}, \rho_{n_{0}} /|\lambda|\right\}$. Let $\alpha(t)=m \lambda(t)$ for $t \in[0, T]_{\mathbb{T}}$. Then $\alpha(t) \in C[0, T]_{\mathbb{T}} \cap C^{\Delta}(0, T]_{\mathbb{T}}, \varphi_{p}\left(\alpha^{\Delta}(t)\right) \in C^{\nabla}(0, T)_{\mathbb{T}}, \alpha(0)=0$ with $0<\alpha(t) \leq \lambda(t) \leq \rho_{n}$ for $t \in\left(1 / 2^{n+1}, T\right]_{\mathbb{T}}$. Without loss of generality, we have $\alpha(T)-\sum_{i=1}^{m} \psi_{i}\left(\alpha^{\Delta}\left(\xi_{i}\right)\right)<0$. For arbitrary $(t, u) \in(0, T]_{\mathbb{T}} \times\left\{u \in C[0, T]_{\mathbb{T}} \cap C^{\Delta}(0, T]_{\mathbb{T}}: 0<u \leq \alpha(t)\right\}$, there exists $n \in\left\{n_{0}, n_{0}+1, \ldots\right\}$ such that $(t, u) \in\left[1 / 2^{n+1}, T\right]_{\mathbb{T}} \times\left\{u \in C[0, T]_{\mathbb{T}} \cap C^{\Delta}(0, T]_{\mathbb{T}}: 0<u \leq \alpha(t)\right\}$. We have

$$
\begin{aligned}
q(t) f(t, u)+\left(\varphi_{p}\left(\alpha^{\Delta}(t)\right)\right)^{\nabla} & \geq k_{0}+\left(\varphi_{p}\left(m \lambda^{\Delta}(t)\right)\right)^{\nabla} \\
& =k_{0}+m^{p-1}\left(\varphi_{p}\left(\lambda^{\Delta}(t)\right)\right)^{\nabla} \\
& \geq k_{0}-m^{p-1}\left|\left(\varphi_{p}\left(\lambda^{\Delta}(t)\right)\right)^{\nabla}\right| \\
& \geq k_{0}-\frac{k_{0}}{2 R_{1}}\left|\left(\varphi_{p}\left(\lambda^{\Delta}(t)\right)\right)^{\nabla}\right| \\
& \geq k_{0}-\frac{k_{0}}{2 R_{1}} \max _{t \in[0, T]}\left|\left(\varphi_{p}\left(\lambda^{\Delta}(t)\right)\right)^{\nabla}\right|=\frac{k_{0}}{2}>0 .
\end{aligned}
$$


Thus (A4) holds and (A2) is also true if $u(t)=\alpha(t)$. Also since $\alpha(T) \leq \sup _{t \in[0, T]_{T}}|\alpha(t)|=$ $m \sup _{t \in[0, T]_{T}}|\lambda| \leq \rho_{n_{0}}$, we have $\beta(T) \geq \rho_{n_{0}} \geq \alpha(T)$, then (A6) is fulfilled. By Corollary 2.3, the boundary value problem (1.1) and (1.2) has a solution $u(t) \in C[0, T]_{\mathbb{T}} \cap C^{\Delta}(0, T]_{\mathbb{T}}, \varphi_{p}\left(u^{\Delta}(t)\right) \in$ $C^{\nabla}(0, T)_{\mathbb{T}}$ with $u(t) \geq 0$ for $t \in(0, T]_{\mathbb{T}}$.

We can replace $t \in\left[1 / 2^{n+1}, T\right]_{\mathbb{T}}$ with $t \in\left[0, T-1 / 2^{n+1}\right]_{\mathbb{T}}$ or $t \in\left[1 / 2^{n+1}, T-1 / 2^{n+1}\right]_{\mathbb{T}}$. So it is easily obtain (see Remark 2.2) the analogue of Theorem 3.2 in this section.

Looking at Theorem 3.2, it is difficulty for us to discuss examples in constructing $\beta(t)$ in (A8). The following theorem removes (A8) and replaces it with an easy verified condition.

Theorem 3.3. Let $n_{0} \in\{1,2, \ldots\}$ be fixed. If (H1)-(H3), (A1) and (A2) hold, in addition suppose that the following conditions are satisfied:

$$
\begin{gathered}
M_{1}>0, \quad M_{2}>\max \left\{\sup _{t \in[0, T]} \alpha(t), \rho_{n_{0}}\right\}, \text { here } M_{1}, M_{2} \text { are constants, } \\
q(t) f\left(t, M_{1} t+M_{2}\right) \leq 0 \quad \text { for } t \in(0, T)_{\mathbb{T}}, \\
q(t) f\left(\frac{1}{2^{n_{0}+1}}, M_{1} t+M_{2}\right) \leq 0 \text { for } t \in\left(0, \frac{1}{2^{n_{0}+1}}\right)_{\mathbb{T}} \\
M_{1} T+M_{2}-\sum_{i=1}^{m} \psi_{i}\left(M_{1}\right)>0 .
\end{gathered}
$$

Then boundary value problem (1.1) and (1.2) has a solution $u \in C[0, T]_{\mathbb{T}} \cap C^{\Delta}(0, T]_{\mathbb{T}}$ with $\varphi_{p}\left(u^{\Delta}\right) \in$ $C^{\nabla}(0, T)_{\mathbb{T}}$ and $u>0$ for $t \in(0, T]_{\mathbb{T}}$.

Proof. Denote $\beta(t)=M_{1} t+M_{2}$ for $t \in[0, T]_{\mathbb{T}}$, then $\beta(t) \in C[0, T]_{\mathbb{T}} \cap C^{\Delta}(0, T]_{\mathbb{T}}, \varphi_{p}\left(\beta^{\Delta}(t)\right) \in$ $C^{\nabla}(0, T)_{\mathbb{T}}$ with $\beta(t) \geq \alpha(t)$ and $\beta(t) \geq \rho_{n_{0}}$ for $t \in[0, T]_{\mathbb{T}}$,

$$
\beta(T)-\sum_{i=1}^{m} \psi_{i}\left(\beta^{\Delta}\left(\xi_{i}\right)\right)>0
$$

with

$$
\begin{gathered}
-\left(\varphi_{p}\left(\beta^{\Delta}(t)\right)\right)^{\nabla} \geq q(t) f(t, \beta) \quad \text { for } t \in(0, T)_{\mathbb{T}}, \\
-\left(\varphi_{p}\left(\beta(t)^{\Delta}(t)\right)\right)^{\nabla} \geq q(t) f\left(\frac{1}{2^{n_{0}+1}}, \beta(t)\right) \quad \text { for } t \in\left(0, \frac{1}{2^{n_{0}+1}}\right)_{\mathbb{T}},
\end{gathered}
$$

then (A3) holds. By Theorem 2.1 the result holds.

From Theorems 3.2 and 3.3 we have the following theorem.

Theorem 3.4. Let $n_{0} \in\{1,2, \ldots\}$ be fixed. If (H1)-(H3), (3.1) and (A7) hold, in addition suppose there exist constants $M_{1}, M_{2}>0$ such that (3.11) and (3.5) are true. Then the problem (1.1) and (1.2) has a solution $u \in C[0, T]_{\mathbb{T}} \cap C^{\Delta}(0, T]_{\mathbb{T}}$ with $\varphi_{p}\left(u^{\Delta}\right) \in C^{\nabla}(0, T)_{\mathbb{T}}$ and $u>0$ for $t \in(0, T]_{\mathbb{T}}$. 
Proof. Without loss of generality suppose $\rho_{n_{0}}<M_{2}$, by (A7) we have (A1) which holds and

$$
M_{2}>\rho_{n_{0}}>\rho_{n_{0}+1}>\ldots, \quad \lim _{n \rightarrow \infty} \rho_{n}=0 .
$$

By the similar way as the proof of the Theorem 3.2, there exists a function $\alpha \in C[0, T]_{\mathbb{T}} \cap$ $C^{\Delta}(0, T]_{\mathbb{T}}, \varphi_{p}\left(\alpha^{\Delta}\right) \in C^{\nabla}(0, T)_{\mathbb{T}}$ with $\alpha(0)=0, \alpha(T)-\sum_{i=1}^{m} \psi_{i}\left(\alpha^{\Delta}\left(\xi_{i}\right)\right)<0, \alpha(t)>0$ for $t \in(0, T]_{\mathbb{T}}$, such that $-\left(\varphi_{p}\left(\alpha^{\Delta}(t)\right)\right)^{\nabla} \leq q(t) f(t, \alpha(t))$ for $t \in(0, T)_{\mathbb{T}}$ and $\alpha(t) \leq \sup _{t \in[0, T]_{\mathbb{T}}}|\alpha| \leq \rho_{n_{0}}$. This together with (3.14) we have $M_{2}>\max \left\{\sup _{t \in[0, T]} \alpha(t), \rho_{n_{0}}\right\}$. Thus all the conditions of the Theorem 3.3 are fulfilled.

\section{An Example}

In this section, we present an example to illustrate our results. Let

$$
\mathbb{T}=\{0\} \cup\left\{\left(\frac{1}{2}\right)^{\mathbb{N}}\right\} \cup\left[\frac{1}{2}, 1\right]
$$

Consider the following boundary value problem

$$
\begin{gathered}
-\left(\left|u^{\Delta}(t)\right|^{2} u^{\Delta}(t)\right)^{\nabla}=q(t) f(t, u(t)) \quad \text { for } t \in(0,1)_{\mathbb{T}}, \\
u(0)=0, \quad u(1)-\frac{1}{5} u^{\Delta}\left(\frac{1}{8}\right)-\frac{1}{10} u^{\Delta}\left(\frac{1}{4}\right)-\frac{1}{5} u^{\Delta}\left(\frac{3}{4}\right)-\frac{1}{10} u^{\Delta}(1)=0 .
\end{gathered}
$$

It is obvious that $T=1, p=4, m=4, \psi_{1}(x)=\psi_{3}(x)=(1 / 5) x, \psi_{2}(x)=\psi_{4}(x)=(1 / 10) x$. Denote $q(t)=t^{8}+5$ and $f(t, u(t))=t / u^{7}(t)+u^{7}(t)-\lambda^{2}$, here $\lambda^{2} \geq 2$ is constant. Let $n_{0} \in\{1,2, \ldots\}, \rho_{n}=$ $\left(1 / 2^{n+1}\left(\lambda^{2}+a_{1}\right)\right)^{1 / 7}$ and $k_{0}=a_{1}>0$ is a constant. We have $\rho_{n_{0}} \leq 1$. Note that (H1)-(H3) and (3.1) hold. For $n \in\{1,2, \ldots\}, t \in\left[1 / 2^{n+1}, 1\right]_{\mathbb{T}}$ and $0<u \leq \rho_{n}$, we have

$$
q(t) f(t, u) \geq\left(t^{8}+5\right)\left(\frac{1}{2^{n+1} \rho_{n}^{7}}-\lambda^{2}\right) \geq\left(t^{8}+5\right)\left(\lambda^{2}+a_{1}-\lambda^{2}\right)>a_{1}
$$

which implies (A7) is satisfied.

Now we show that (A8) holds with $\beta=t^{1 / 7}$.

Notice that if $t \in(1 / 2,1]_{\mathbb{T}}$, then $\beta^{\Delta}(t)=\beta^{\prime}(t)=(1 / 7) t^{-6 / 7}$,

$$
\begin{gathered}
\left|\beta^{\Delta}(t)\right|^{2} \beta^{\Delta}(t)=\frac{1}{343} t^{-18 / 7}, \quad\left(\left|\beta^{\Delta}(t)\right|^{2} \beta^{\Delta}(t)\right)^{\nabla}=-\frac{18}{2401} t^{-25 / 7} \leq 0 . \\
\text { If } t=1 / 2 \text {, then } \beta^{\Delta}(t)=(1 / 7) t^{-6 / 7} \text { and }\left|\beta^{\Delta}(t)\right|^{2} \beta^{\Delta}(t)=(1 / 343) t^{-18 / 7}, \\
\left(\left|\beta^{\Delta}(t)\right|^{2} \beta^{\Delta}(t)\right)^{\nabla}=\frac{2}{343}\left(\frac{1}{2}\right)^{-18 / 7}-\frac{2}{343}\left(\frac{1}{4}\right)^{-18 / 7} \approx-0.1714 \leq 0 .
\end{gathered}
$$




$$
\begin{aligned}
& \text { If } t=1 / 2^{n}(n=2,3, \ldots) \text {, then } \sigma(t)=2 t, \rho(t)=t / 2, \mu(t)=t, v(t)=t / 2 \text {, we have } \\
& \qquad \beta^{\Delta}(t)=\frac{1}{t}\left[(2 t)^{1 / 7}-t^{1 / 7}\right], \quad\left|\beta^{\Delta}(t)\right|^{2} \beta^{\Delta}(t)=\frac{1}{t^{3}}\left[(2 t)^{1 / 7}-t^{1 / 7}\right]^{3},
\end{aligned}
$$

by induction, one gets

$$
\left(\left|\beta^{\Delta}(t)\right|^{2} \beta^{\Delta}(t)\right)^{\nabla}=2^{4 n+1}\left[\left(\frac{1}{2^{n-1}}\right)^{1 / 7}-\left(\frac{1}{2^{n}}\right)^{1 / 7}\right]^{3}-2^{4 n+4}\left[\left(\frac{1}{2^{n}}\right)^{1 / 7}-\left(\frac{1}{2^{n+1}}\right)^{1 / 7}\right]^{3} \leq 0 .
$$

Thus, for $t \in(0,1)_{\mathbb{T}}$, we have

$$
\begin{aligned}
\left(\left|\beta^{\Delta}(t)\right|^{2} \beta^{\Delta}(t)\right)^{\nabla}+q(t) f(t, \beta) & \leq\left(t^{8}+5\right)\left(\frac{t}{t}+\left(t^{1 / 7}\right)^{7}-\lambda^{2}\right) \\
& \leq\left(t^{8}+5\right)\left(2-\lambda^{2}\right) \leq 0 \\
\left(\left|\beta^{\Delta}(t)\right|^{2} \beta^{\Delta}(t)\right)^{\nabla}+q(t) f\left(\frac{1}{2^{n_{0}+1}}, \beta\right) & \leq\left(t^{8}+5\right)\left(\frac{1}{2^{n_{0}+1}\left(t^{1 / 7}\right)}+\left(t^{1 / 7}\right)^{7}-\lambda^{2}\right) \\
& \leq\left(t^{8}+5\right)\left(2-\lambda^{2}\right) \leq 0 \quad \text { for } t \in\left(0, \frac{1}{2^{n_{0}+1}}\right)_{\mathbb{T}}
\end{aligned}
$$

Now

$$
\beta(1)-\psi_{1}\left(\beta^{\Delta}\left(\frac{1}{8}\right)\right)-\psi_{2}\left(\beta\left(\frac{1}{4}\right)\right)-\psi_{3}\left(\beta^{\Delta}\left(\frac{3}{4}\right)\right)-\psi_{4}(\beta(1))>0
$$

Hence, all conditions of the Theorem 3.2 are satisfied. As a result, the problem (4.2) has a positive solution.

\section{Acknowledgments}

This paper is supported by XZIT under Grant XKY2008311 and DEGP under Grant 0709-03.

\section{References}

[1] S. Hilger, Ein Maßkettenkalkül mit Anwendung auf Zentrumsmannigfaltigkeiten, Ph.D. thesis, Universität Würzburg, Würzburg, Germany, 1988.

[2] R. P. Agarwal, M. Bohner, and P. Řehák, "Half-linear dynamic equations," in Nonlinear Analysis and Applications: to V. Lakshmikantham on His 80th Birthday. Vol. 1, pp. 1-57, Kluwer Academic Publishers, Dordrecht, The Netherlands, 2003.

[3] M. Bohner and A. Peterson, Dynamic Equations on Time Scales: An Introduction with Applications, Birkhäuser, Boston, Mass, USA, 2001.

[4] M. A. Jones, B. Song, and D. M. Thomas, "Controlling wound healing through debridement," Mathematical and Computer Modelling, vol. 40, no. 9-10, pp. 1057-1064, 2004. 
[5] V. Spedding, "Taming nature's numbers," New Scientist, no. 2404, pp. 28-32, July 2003.

[6] D. M. Thomas, L. Vandemuelebroeke, and K. Yamaguchi, "A mathematical evolution model for phytoremediation of metals," Discrete and Continuous Dynamical Systems. Series B, vol. 5, no. 2, pp. 411-422, 2005.

[7] M. Bohner and A. Peterson, Advances in Dynamic Equations on Time Scales, Birkhäuser, Boston, Mass, USA, 2003.

[8] B. Aulbach and L. Neidhart, "Integration on measure chains," in Proceedings of 6th International Conference on Difference Equations, pp. 239-252, CRC, Augsburg, Germany, July-August 2004.

[9] C. J. Chyan and P. J. Y. Wong, "Multiple positive solutions of conjugate boundary value problems on time scales," Taiwanese Journal of Mathematics, vol. 11, no. 2, pp. 421-445, 2007.

[10] J. Hoffacker, "Green's functions and eigenvalue comparisons for a focal problem on time scales," Computers \& Mathematics with Applications, vol. 45, no. 6-9, pp. 1339-1368, 2003.

[11] J. Hoffacker and C. C. Tisdell, "Stability and instability for dynamic equations on time scales," Computers \& Mathematics with Applications, vol. 49, no. 9-10, pp. 1327-1334, 2005.

[12] D. R. Anderson, G. Sh. Guseinov, and J. Hoffacker, "Higher-order self-adjoint boundary-value problems on time scales," Journal of Computational and Applied Mathematics, vol. 194, no. 2, pp. 309 342, 2006.

[13] W.-T. Li and X.-L. Liu, "Eigenvalue problems for second-order nonlinear dynamic equations on time scales," Journal of Mathematical Analysis and Applications, vol. 318, no. 2, pp. 578-592, 2006.

[14] Y.-H. Su and W.-T. Li, "Triple positive symmetric solutions of $p$-Laplacian BVPs on time scales," Acta Mathematica Sinica, Chinese Series, vol. 52, pp. 181-196, 2009.

[15] Y.-H. Su, X. H. Yuan, and X.-X. Yan, "Existence of solution to a three-point BVPs for $p$-Laplacian dynamic equations on time scales," Journal of Lanzhou University, Natural Sciences, vol. 44, pp. 112116, 2008.

[16] J.-P. Sun, "Existence of solution and positive solution of BVP for nonlinear third-order dynamic equation," Nonlinear Analysis: Theory, Methods \& Applications, vol. 64, no. 3, pp. 629-636, 2006.

[17] D. Anderson, R. Avery, and J. Henderson, "Existence of solutions for a one dimensional $p$-Laplacian on time-scales," Journal of Difference Equations and Applications, vol. 10, no. 10, pp. 889-896, 2004.

[18] Z. He, "Double positive solutions of three-point boundary value problems for $p$-Laplacian dynamic equations on time scales," Journal of Computational and Applied Mathematics, vol. 182, no. 2, pp. 304-315, 2005.

[19] Y.-H. Su, "Multiple positive pseudo-symmetric solutions of $p$-Laplacian dynamic equations on time scales," Mathematical and Computer Modelling, vol. 49, no. 7-8, pp. 1664-1681, 2009.

[20] Y.-H. Su and W.-T. Li, "Triple positive solutions of $m$-point BVPs for $p$-Laplacian dynamic equations on time scales," Nonlinear Analysis: Theory, Methods E Applications, vol. 69, no. 11, pp. 3811-3820, 2008.

[21] Y.-H. Su, W.-T. Li, and H.-R. Sun, "Triple positive pseudo-symmetric solutions of three-point BVPs for $p$-Laplacian dynamic equations on time scales," Nonlinear Analysis: Theory, Methods E Applications, vol. 68, no. 6, pp. 1442-1452, 2008.

[22] H.-R. Sun and W.-T. Li, "Existence theory for positive solutions to one-dimensional $p$-Laplacian boundary value problems on time scales," Journal of Differential Equations, vol. 240, no. 2, pp. $217-$ 248, 2007.

[23] Y.-H. Su and W.-T. Li, "Existence of positive solutions to a singular $p$-Laplacian dynamic equations with sign changing nonlinearity," Acta Mathematica Sinica, Chinese Series, vol. 28, pp. 51-60, 2008.

[24] Y.-H. Su, W.-T. Li, and H.-R. Sun, "Positive solutions of singular $p$-Laplacian dynamic equations with sign changing nonlinearity," Applied Mathematics and Computation, vol. 200, no. 1, pp. 352-368, 2008.

[25] Y.-H. Su, W.-T. Li, and H.-R. Sun, "Positive solutions of singular $p$-Laplacian BVPs with sign changing nonlinearity on time scales," Mathematical and Computer Modelling, vol. 48, no. 5-6, pp. 845-858, 2008.

[26] R. P. Agarwal, H. Lü, and D. O'Regan, "Existence theorems for the one-dimensional singular $p$ Laplacian equation with sign changing nonlinearities," Applied Mathematics and Computation, vol. 143, no. 1, pp. 15-38, 2003.

[27] D. O'Regan, “Upper and lower solutions for singular problems arising in the theory of membrane response of a spherical cap," Nonlinear Analysis: Theory, Methods E Applications, vol. 47, no. 2, pp. 1163-1174, 2001.

[28] H. Lü, D. O’Regan, and R. P. Agarwal, "Existence theorems for the one-dimensional singular $p$ Laplacian equation with a nonlinear boundary condition," Journal of Computational and Applied Mathematics, vol. 182, no. 1, pp. 188-210, 2005. 
[29] H. Lü, D. O’Regan, and R. P. Agarwal, “Upper and lower solutions for the singular $p$-Laplacian with sign changing nonlinearities and nonlinear boundary data," Journal of Computational and Applied Mathematics, vol. 181, no. 2, pp. 442-466, 2005.

[30] D. Q. Jiang, D. O’Regan, and R. P. Agarwal, “A generalized upper and lower solution method for singular discrete boundary value problems for the one-dimensional p-Laplacian," Journal of Applied Analysis, vol. 11, no. 1, pp. 35-47, 2005.

[31] V. Lakshmikantham, S. Sivasundaram, and B. Kaymakcalan, Dynamic Systems on Measure Chains, vol. 370 of Mathematics and Its Applications, Kluwer Academic Publishers, Dordrecht, The Netherlands, 1996. 


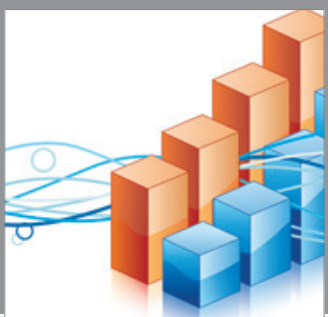

Advances in

Operations Research

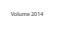

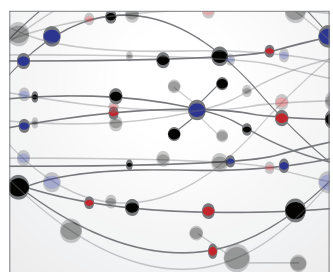

\section{The Scientific} World Journal
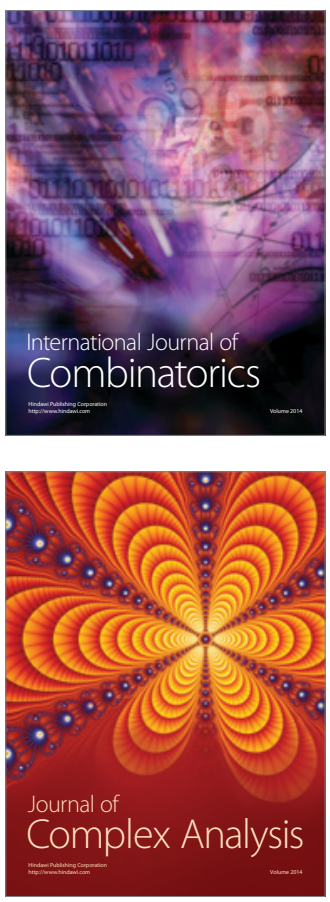

International Journal of

Mathematics and

Mathematical

Sciences
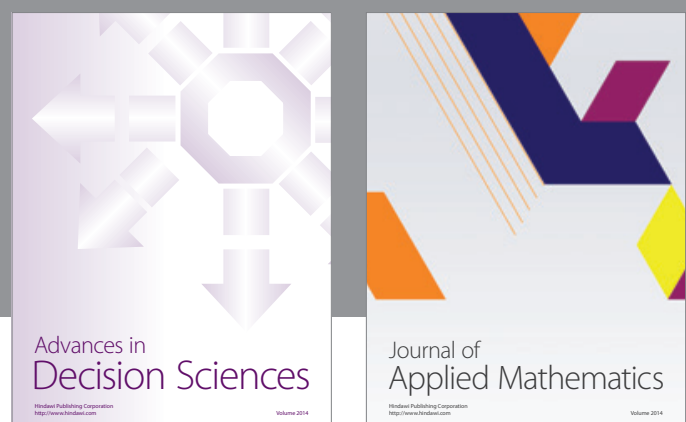

Journal of

Applied Mathematics
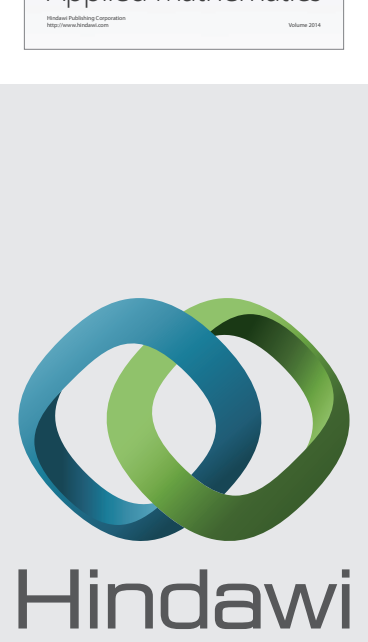

Submit your manuscripts at http://www.hindawi.com
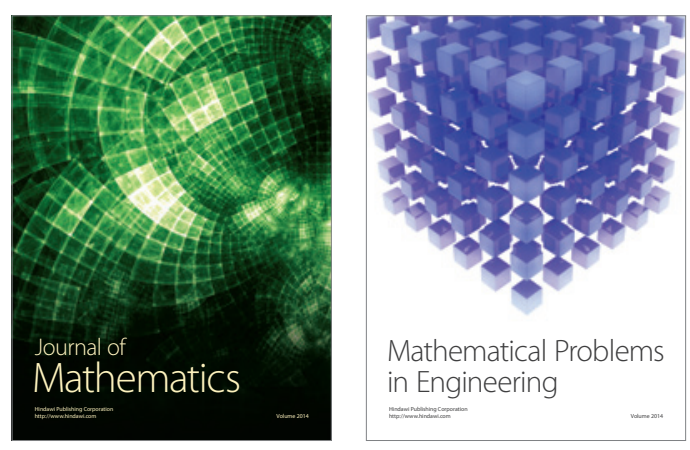

Mathematical Problems in Engineering
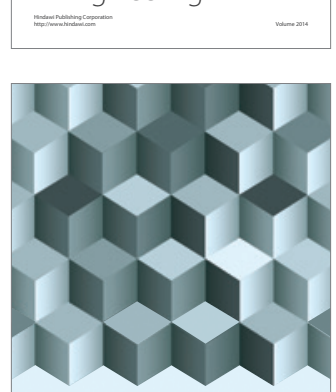

Journal of

Function Spaces
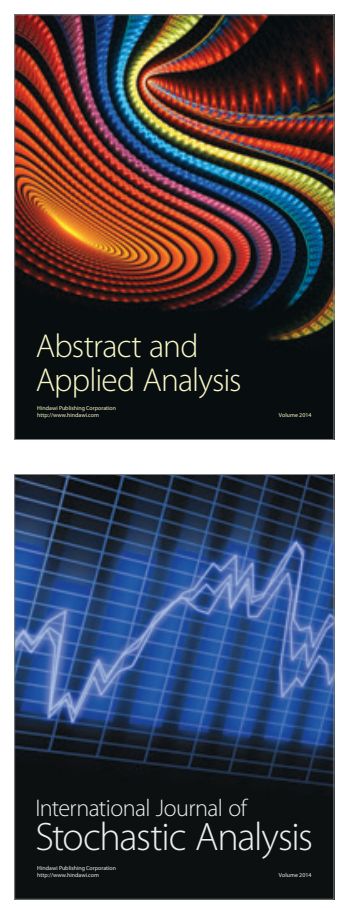

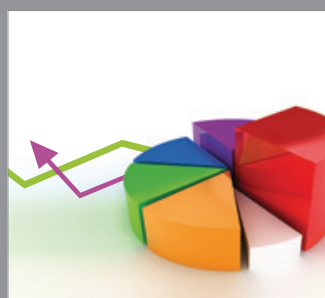

ournal of

Probability and Statistics

Promensencen
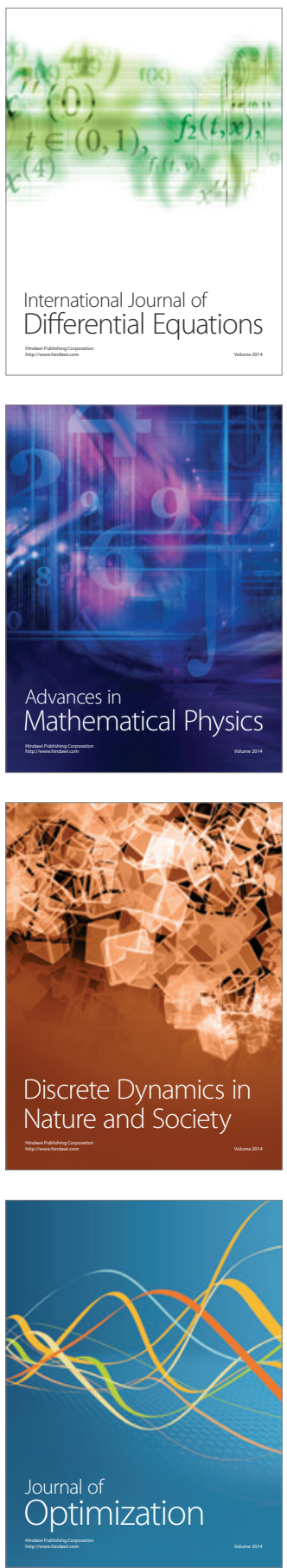